\title{
Dynamic causal modeling of touch-evoked potentials in the rubber hand illusion
}

Daniel Zeller ${ }^{1}$, Karl J. Friston ${ }^{2}$, and Joseph Classen ${ }^{1,3}$

1) Dept. of Neurology, University of Würzburg, 97080 Würzburg, Germany

2) The Wellcome Trust Centre for Neuroimaging, UCL Institute of Neurology, Queen Square, London WC1N 3BG, UK

3) Dept. of Neurology, University of Leipzig, 04103 Leipzig, Germany

Running Title: RHI \& DCM

Number of figures: 3

Number of tables: 1

Word count (abstract): $200(<=200)$

Word count (main text): 4.071

\section{Corresponding author:}

Prof. J. Classen, MD

Dept. of Neurology, University of Leipzig

Liebigstr. 20, 04103 Leipzig, Germany

Tel. ++49 (0)3419724200 / Fax. ++49 (0)3419724209

e-mail: joseph.classen@medizin.uni-leipzig.de 


\section{Abstract}

The neural underpinnings of bodily ownership can be disclosed by the rubber hand illusion (RHI), which refers to the illusory self-attribution of an artificial hand induced by synchronous tactile stimulation of a subject's hidden hand and a visible artificial hand. To investigate the effective connectivity between and within brain areas involved in generating bodily perceptions we applied dynamic causal modeling to touch-evoked responses in 13 healthy subjects whose right hand was stroked while viewing their own hand ("REAL"), or an artificial hand presented in an anatomically plausible ("CONGRUENT") or impossible ("INCONGRUENT") position. Bayesian model comparison revealed strong evidence for a differential involvement of premotor cortex (PMC) in the generation of touch-evoked responses under the three conditions, confirming a crucial role of PMC in bodily self-attribution: the forward connection from left occipital cortex to PMC was stronger for CONGRUENT and INCONGRUENT as compared to REAL, illustrating the importance of bottom-up influence of visual input when multisensory integration is challenged. Crucially, intrinsic connectivity in $\mathrm{S} 1$ was reduced in the CONGRUENT condition which was tied to the perception of a RHI. These findings support predictive coding models of the functional architecture of multisensory integration in dynamic bodily perceptual experience.

Keywords: bodily ownership, multisensory integration, predictive coding, premotor cortex, touch-evoked potentials. 


\section{INTRODUCTION}

In everyday life, we commonly experience situations where there is more than one explanation for the origin of our sensations. For example, if we are sitting in a train and the train next to ours starts pulling out, we might experience that it is either our train or the other that is moving. When we listen to a ventriloquist, we might perceive the voice to emulate from the speaker's motionless mouth or from the dummy's moving mouth. In such circumstances, the brain needs to weigh competing hypotheses in order to select one plausible explanation that constitutes our percept, thereby satisfying the strong human need to make sense of the world around oneself.

Different perceptual illusions have been used experimentally to unveil the basic principles of how the brain resolves conflicting multisensory input (e. g. \{Ehrsson, 2004 \#105;Zeller, 2011 \#292;Guterstam, 2011 \#814;Petkova, 2008 \#815;Ehrsson, 2007 \#819\}). One powerful experimental paradigm is the "rubber hand illusion" (RHI), which refers to the induction of a feeling of limb-ownership by synchronous tactile stimulation of a subject's hidden hand and a visible plastic hand \{Botvinick, $1998 \# 98$ \}. The RHI taps into the neural underpinnings of our feeling of bodily self and its notable malleability \{Armel, 2003 \#293\}. Furthermore, it represents an interesting example for inference in the setting of multisensory integration $\{$ Hohwy, 2010 \#402\}: Initially, the brain has access to proprioceptive information about the position of the subject's hand, but also visual information about the location of the seen touch. In addition, there is mechanoreceptive information about the touch. Since the proprioceptive and the visual sensory inputs are conflicting, the brain has the resolve uncertainty about the real position of one's own hand. One solution could be to select one hypothesis and ignore the other (which is commonly referred to as sensory dominance); another is to make a combined estimate halfway between the unimodal estimates \{O'Reilly, $2012 \# 809\}$. An intuitive third solution might be to consider both inputs at once, but weight them by their relative precisions - in other words, to consider each 
measurement with the appropriate level of confidence \{O'Reilly, $2012 \# 809$;Brown, $2013 \# 323$ \}. This Bayesian approach to the problem of contradictory or ambiguous sensory inputs is a core element of the framework provided by predictive coding \{Friston, 2008 \#385;Friston, 2007 $\# 386$ \}. According to this framework, the brain constantly interprets sensory information under a hierarchical generative model of the world \{Limanowski, $2015 \# 857\}$. Any mismatch between predicted and actual sensory input generates prediction errors which are referred to the level above by bottom-up (forward or ascending) connections. In order to minimize prediction errors, high-level expectations are updated so that top-down (backward or descending) predictions “explain away" the prediction error \{Limanowski, $2015 \# 857\}\{$ Murray, $2002 \# 858$ \}. Based on this predictive coding account, we have previously suggested that RHI emerges through attenuation of somatosensory precision. We conjectured that to optimally accommodate the uncertainty resulting from conflicting inputs, the influence of ascending somatosensory prediction errors on top-down predictions would be attenuated by reducing their precision \{Zeller, $2014 \# 810\}$. In line with this hypothesis, we found that touch-evoked potentials elicited by brush-strokes were specifically attenuated during the RHI \{Zeller, 2014 \#810\}. However, the question which causal functional connections at the network level led to attenuation of cortical responses remained open.

Within the framework provided by predictive coding, a change in sensory precision should translate into intrinsic modulation in S1 and/or modulation of forward connections between somatosensory input regions and higher-level multimodal regions - the latter of thought to be located within the ventral premotor cortex (PMC) and the parietal cortex \{Ehrsson, $2004 \# 105$ \}. To test this hypothesis, we used dynamic causal modeling (DCM) of electroencephalographic (EEG) data \{David, $2006 \# 811\}$ followed by family level inference $\{$ Penny, $2010 \# 813$ \}. DCM uses a biologically informed neuronal network model to make inferences about the underlying 
(effective or directed) connectivity generating evoked responses \{David, $2006 \# 811$ \}. The parameters of this neurobiologically constrained source reconstruction scheme have an explicit neuronal interpretation - they include information about the coupling among sources and how that coupling depends upon the experimental context \{David, $2006 \# 811\}$. Based on early touchevoked potentials elicited by the brushstrokes \{Zeller, 2014 \#810\}, we applied Bayesian model comparison to identify those differences in coupling among (extrinsic) and within (intrinsic) sources of the touch-evoked responses that distinguish between the illusory feeling of ownership of a plastic hand and two control conditions. We hypothesized that DCM would reveal (i) involvement of the ventral PMC and its (extrinsic) connections, and (ii) attenuation of (intrinsic) connectivity within S1. 


\section{METHODS}

\section{Subjects}

Thirteen healthy volunteers ( 9 men, 4 women; 10 right handed, 3 ambidextrous) aged 21 to 32 years (mean $24.7 \pm 4.1$ years) were included. Evoked potential data from this group of volunteers have been published previously $\{$ Zeller, $2014 \# 810\}$. As described there $\{$ Zeller, $2014 \# 810\}$, all subjects were familiar with the RHI setting and were known to experience the RHI, but were naïve to the particular purpose of this study. The study was approved by the Ethics committee of the Medical Faculty at the University of Würzburg and conformed to the principles of the declaration of Helsinki. All volunteers gave their written informed consent for this research study.

\section{EEG recording}

EEG signals were recorded from 64 surface electrodes placed at extended 10-20 system locations and fixed on the subject's head with an elastic cap (EASYCAP GmbH, Herrsching-Breitbrunn, Germany). The electrode impedance was kept below $5 \mathrm{k} \Omega$. The brain signals were recorded with a Quickamp system (Brain Products GmbH, Munich, Germany), average referenced in hardware, filtered between 0 and $560 \mathrm{~Hz}$, and sampled at $2000 \mathrm{~Hz}$. Three-dimensional electrode positions and anatomic reference points were registered using an ultrasound-based navigation system (Zebris Medical GmbH, Isny, Germany).

\section{Experimental setup}

The setup has been described in detail previously \{Zeller, 2014 \#810\}. Briefly, the subject's right hand received repetitive brushstrokes in the following three conditions: (i) REAL: Hand and forearm were resting on the table. The eyes were fixating on the real hand, which was repetitively 
touched by a brush as described below. (ii) CONGRUENT: The subject's real hand was hidden from view in a shoebox with a $10 \mathrm{~cm} \times 8 \mathrm{~cm}$ open window on both sides. A realistic, life-sized, gender- and side-matched artificial hand was placed on the top of the box in an anatomically plausible position. The gap between the trunk of the artificial hand and the subject's upper arm was covered with a towel. The eyes fixated on the artificial hand while the experimenter used two interconnected small paintbrushes to stroke the (visible) artificial hand and the (hidden) real hand as synchronously as possible. (iii) INCONGRUENT: The artificial hand was flipped $180^{\circ}$ around its longitudinal axis (palm upwards) with an otherwise identical setting as described for

\section{CONGRUENT.}

In all conditions, small and brisk brushstrokes were applied to the dorsal surface of the middle phalanx of the index, middle, and ring finger at 0.5 to $3 \mathrm{~Hz}$. Touches were registered as trigger signals by a custom-built brush handle containing an electrical switch for subsequent analysis of touch evoked potentials. Tactile stimulation was applied in six blocks of 5 min each (two blocks per condition, randomized order). During continuous EEG recording, a second experimenter set markers indicating the respective stroking condition. By pressing a foot switch, subjects indicated whether the feeling that the rubber hand was their own hand occurred (one press) or disappeared (two presses) in the CONGRUENT and INCONGRUENT conditions.

\section{Evoked potential analysis}

Data preparation: Our previous study had revealed significant differences of touch evoked potentials between the three stimulation conditions for right hand stimulation \{Zeller, 2014 $\# 810$ \}. Hence, data from the right hand side was used for further analysis. The triggers were sorted based on the stimulation condition and the presence or absence of the illusion. Individual EEG data were epoched between -200 and +300 ms relative to the touch trigger signal. Trials 
were triaged for artefacts and rejected if they met one of the following conditions: 1) The amplitude difference between the largest and the smallest sample within the trial in any channel exceeded $200 \mu \mathrm{V}$. 2) The voltage difference between two adjacent samples in any channel exceeded $75 \mu \mathrm{V}$.

Averages were computed using BESA software (BESA GmbH, Gräfelfing, Germany). As reported earlier \{Zeller, $2014 \# 810$ \}, subjects reported the presence of the illusion in $85.5 \%$ (median; range $43 \%$ to 100\%) of the epochs during CONGRUENT and absence of the illusion in 93.0\% (median; range $25 \%$ to $100 \%$ ) epochs during INCONGRUENT. Therefore, as sufficient numbers of epochs are required for averaging, evoked potentials were obtained under three conditions: real hand stimulation only (REAL), synchronous stimulation in an anatomically plausible position of the artificial hand and induction of the RHI (CONGRUENT), and synchronous stimulation with an anatomically impossible position of the artificial hand and absence of the RHI (INCONGRUENT). We used SPM12 software for all subsequent steps of data analysis (Litvak et al., 2011; www.fil.ion.ucl.ac.uk/spm). The average responses were bandpass filtered between 2 and $20 \mathrm{~Hz}$ to establish a flat baseline and remove high-frequency noise.

$3 D$ source reconstruction: To obtain source location priors for subsequent DCM analysis, we used the 3D source reconstruction routine of SPM 12. Grand average data of all subjects across all stimulation conditions were used, with additional parameters as follows: template: fine (mesh); forward model: EEG BEM; reconstruction: imaging; standard model; time window: 0 to 250 ms relative to the touch trigger signal; all frequencies.

DCM specification: We considered dynamic causal models that either include or do not include bilateral ventral premotor cortex (PMC) or parietal cortex (PC), respectively (Fig. 1A). The MNI coordinates $(\mathrm{x}, \mathrm{y}, \mathrm{z})$ of these cortical areas were obtained from \{Ehrsson, $2004 \# 105$ \}: right PMC 
$(48,18,39)$, left PMC $(-57,15,9)$, right intraparietal cortex $(33,-45,51)$, and left intraparietal cortex $(-36,-42,51)$. In addition, the postcentral gyrus (S1) contralateral to the stimulated hand and bilateral occipital regions (OC), which were identified by 3D source reconstruction, were considered as somatosensory and visual input regions, respectively: left $\mathrm{S} 1(-38,-31,55)$, right middle occipital gyrus $(28,-62,39)$, and left middle occipital gyrus $(-28,-62,39)$.

Our extrinsic effective connectivity was based on the assumption of (i) reciprocal connections between each of the three input regions with the ipsilateral PMC or PC, respectively \{Rizzolatti, 1998 \#828;Brozzoli, $2012 \# 821$ \}, and (ii) transcallosal connections between homologous cortical regions (PMC, PC, OC) \{Hofer, $2006 \# 829\}$. Importantly, whereas all the sources in the PMC or PC models were reciprocally connected with extrinsic connections (Fig. 1A), each of the 32 models considered only allowed a subset of connections to change under each experimental condition (Fig. 1B). Additionally, intrinsic connectivity changes within the cortical input sources were considered, given our hypotheses about sensory attenuation.

For DCM analysis, seven sources (left and right PMC, left and right PC, left S1, left and right OC), were modeled using patches on the cortical surface (option "IMG" in the electromagnetic model specification), which were a priori positioned at the MNI coordinates specified above. Sources were interconnected by forward, backward, and lateral connections as illustrated in Fig. 1A to produce two sets of DCMs connecting PMC or PC to the other sources. Two between-trial effects of interest were specified: CONGRUENT-REAL and INCONGRUENT-REAL. These model the effects of experimental condition by modulating the connections specified in each DCM (Fig. 1B). While differences between CONGRUENT and REAL entail the perception of an artificial hand as belonging to oneself (i.e., the illusion), the differences between INCONGRUENT and REAL refer to the visual perception of an artificial hand without an associated feeling of ownership. 
Additional DCM parameters were: time window -50 to $150 \mathrm{~ms}$, one discrete cosine transform term to remove low frequency drifts, no down-sampling, spatial projection of channel data onto eight modes (i.e. eight principal components; see \{Fastenrath, 2009 \#839\} for detailed explanation) for data reduction $\{$ Litvak, $2011 \# 320\}$, stimulus onsets at $0 \mathrm{~ms}$ for $\mathrm{S} 1$ and $0 \mathrm{~ms}$ for bilateral OC. We used the standard 'ERP' neural mass model.

Family level Bayesian model comparison: A total of 32 models were fit to the grand average data of thirteen subjects. Model evidence at the family level was estimated using a fixed effects analysis (FFX) of the negative free energy obtained for every model \{Stephan, 2009 \#812;Penny, $2010 \# 813$ \}. In DCM, the negative free energy $\mathrm{F}$ is the objective function for model inversion. It basically reflects the difference between two opposing requirements of a good model: that it explains the data and is as simple as possible \{Stephan, $2007 \# 841$ \}. Five pairs of families were defined: PMC vs. PC, present vs. absent modulation of S1 forward connections, present vs. absent modulation of OC forward connections, presence vs. absence of S1 intrinsic modulation, and presence vs. absence of OC intrinsic modulation (Fig. 1B and Table 1). Family level inference accommodates uncertainty about aspects of model structure other than the characteristic of interest $\{$ Penny, $2010 \# 813\}$. The advantage of this approach is that - similar to factorial experimental designs - all models are used to answer a number of (usually orthogonal) questions \{Penny, $2010 \# 813\}$. If, for instance, the family „PMC“ (containing models with participation of PMC) exceeds the complementary family „PC“, this indicates that PMC and its connections are more relevant for explaining evoked responses and differences among conditions than PC.

The choice of model families was motivated by our hypotheses that the induction of the RHI relies on a change in sensory precision, which should translate into an attenuation of connectivity 
within S1 and/or modulation of forward connections between input and multimodal regions, and crucially involves the ventral PMC and its connections. 


\section{RESUlts}

All subjects were able to complete the experiment without difficulty. Touch-evoked potentials were recorded as an initially-positive wave with a maximum over the posterior contralateral part of the scalp, along with an initially-negative wave with its maximum over the frontocentral part, both peaking at around $50 \mathrm{msec}$ \{Zeller, $2014 \# 810\}$.

\section{Dynamic causal modelling}

Bayesian model comparison at the family level revealed a strong preference for models containing the PMC, modulation of forward connections from OC, and intrinsic modulation in S1, but lacking modulation of forward connections from S1 and intrinsic modulation in OC (Fig. 2A). Accordingly, when the family exceedance probabilities were compared at the level of all included models, model $\mathrm{N}^{\mathrm{o}} 6$ far exceeded the remaining 31 models (Fig. 2B). The exceedance probability of this model indicates that the main differences among stimulation conditions were mediated by changes in inter-areal connectivity between OC and PMC, and by intrinsic connectivity changes in the left $\mathrm{S} 1$.

We subsequently looked at the winning model $N^{\circ} 6$ (Fig. 3B) for the quantitative (parametric) effects that explain the different responses during the three conditions REAL, CONGRUENT, and INCONGRUENT. While there were no significant connectivity changes in the forward connection from the right $\mathrm{OC}$ to the right PMC, the forward connection from the left OC to the left PMC was significantly stronger for the CONGRUENT (91.06\%; posterior probability $=0.95)$, and even stronger for the INCONGRUENT $(134.63 \%$; posterior probability $=1.00)$, condition as compared to REAL (81.57\%; Fig. 3C).

The intrinsic connectivity of left S1 was significantly lower for the CONGRUENT (97.66\%; posterior probability $=1.00)$ than for the REAL $(102.47 \%)$ condition whereas it was comparable 
between INCONGRUENT and REAL (Fig. 3A). In the neuronal mass model used in this DCM, this corresponds to an attenuation of postsynaptic sensitivity \{Kiebel, $2007 \# 856\}$. 


\section{DISCUSSION}

Based on dynamic causal modeling of EEG data and Bayesian model comparison, this study investigated the differential modulation of both extrinsic connections and intrinsic connectivity during the RHI. We found strong evidence for involvement of the PMC in explaining responses elicited by synchronous brush-strokes in conditions differing in the attribution of bodily self to a fake hand. Moreover, the perception of the RHI was associated with an increase of connectivity between the left OC and left PMC, and a decrease of intrinsic connectivity in the left S1. These results are consistent with predictive coding formulations of the rubber hand illusion. Put simply, during illusory perception, greater precision is afforded to visual input (from the OC) by the PMC, which (we suppose) attenuates the precision of conflicting proprioceptive (limb position) signals through top-down modulation of intrinsic connectivity in S1. Psychologically, this corresponds to attending to visual cues (Feldman and Friston, 2010), while attenuating incompatible proprioceptive cues, to select a plausible (if illusory) perceptual hypothesis - a hypothesis that accounts for synchronous somatosensory and visual information that would be difficult for the brain to explain otherwise.

\section{Role of premotor cortex in bodily self-attribution}

Bayesian model selection clearly favored the family of models with the PMC rather than the PC mediating the differences among the three conditions. This finding agrees with observations that although parietocerebellar areas are active in RHI, the subjective rating of the illusion and the level of neural activity are significantly correlated, specifically in the PMC \{Ehrsson, 2004 $\# 105\}\{$ Brozzoli, $2012 \# 821\}\{$ Gentile, $2013 \# 483$ \}. In support of a causal (as opposed to merely associated) involvement of the PMC, we have previously provided evidence to suggest that ischemic lesions affecting connections to/from the ventral PMC may impair the embodiment of 
an artificial hand as tested by the RHI paradigm \{Zeller, 2011 \#292\}. Our findings, therefore, agree with various lines of behavioral as well as anatomical evidence supporting a key role of the ventral PMC in the generation of the sense of ownership for the artificial hand.

\section{Occipital-premotor connectivity vs. parietal-premotor connectivity in bodily self-attribution}

We found a significant increase of forward connectivity between the left OC and left PMC in the CONGRUENT as compared to the REAL condition. Interestingly, although the pPC is known to integrate unimodal inputs related to mobile body parts into stable spatial representations $\{$ Serino, $2013 \# 832$ \}, the connection between OC and PC did not emerge from the analysis. This does not support a key role of PC in differential processing between conditions, in particular between CONGRUENT and REAL. It also appears to exclude a role of the PC as a major relay node for increased occipito-premotor connectivity, as in this case, one would expect a co-instantaneous increase of forward connectivity between OC and PC. Exactly by which anatomical relays, like prefrontal or subcortical nodes \{Yamagata, 2009 \#848\}\{Dancause, 2006 \#850\}, occipitalpremotor connectivity is mediated must remain speculative and is beyond the scope of this study. The increase of forward connectivity was confined to the left $\mathrm{OC}$ and PMC, i.e. contralateral to the stimulated hand, but did not appear ipsilaterally. This is plausible in view of the experimental setup: Brushstrokes were applied to the right hand along with visual fixation of this hand. A seminal physiological study in non-human primates has shown that bimodal (tactile and visual) cells in the ventral premotor cortex (ventral area 6 of the macaque) - with tactile receptive fields on the arm - encode the space near the body in arm-centered rather than retinocentric coordinates \{Graziano, 1994 \#852\}. This implies a major involvement of the left PMC in multisensory representation of the right arm. Less likely, the observed asymmetry reflects a left-hemispheric lateralization of the premotor capacity for multisensory integration. 
Stronger forward connectivity between the left OC and left PMC points to increased bottom-up influence of the visual input node onto the PMC, i. e. onto the singular multisensory node to which we attribute the occurrence of the illusionary feeling of hand ownership. From a Bayesian perspective, higher precision of visual information, relative to proprioceptive input, should result in increased bottom-up influence on the PMC. At first, it may seem odd that the left OC and PMC showed stronger connectivity not only in the CONGRUENT, but also in the INCONGRUENT condition as compared to REAL. This appears to suggest that this connectivity bears no direct relationship with the illusion of ownership which was present in CONGRUENT, but absent in INCONGRUENT. These two conditions differ by a lack of anatomical plausibility of the position of the artificial hand, while there is the same degree of temporal synchronicity of brushstrokes and the same spatial separation of the real and the plastic hand. In other words, rather than being categorically different, the two conditions vary in the degree of intermodal discrepancy between proprioceptive and visual input - but the percepts induced in both conditions rely upon precise visual information. We consider it likely that the prior predictions of our subjects about the sensory consequences of the INCONGRUENT setup had already been influenced by earlier experiences with the canonical RHI. Expecting the perception of ownership, which turns out to be virtually impossible in INCONGRUENT, may cause a considerable increase in bottom-up influence of (behaviorally speaking: attention to) the visual input which already had been rated trustable earlier. The strong increase of the forward connection between the left OC and PMC in the INCONGRUENT condition may be the physiological correlate of this attentional set.

A recent study has employed DCM on functional imaging data acquired during induction of the RHI and a control condition with asynchronous brushstrokes. The authors describe a significant increase of connections from the lateral occipitotemporal complex and the secondary 
somatosensory cortex to the intraparietal sulcus under synchronous versus asynchronous stimulation \{Limanowski, $2015 \# 857$ \}. They conclude that ambiguous sensory input about one's body configuration may result in the generation of prediction errors in visual and somatosensory areas, which may be conveyed to parietal integrative areas \{Limanowski, $2015 \# 857\}$. However, because several features, including experimental design, imaging method and analysis, distinguish the present study from that by Limanowski and co-workers \{Limanowski, 2015 \#857\} direct comparison of the results is difficult. While the increase of forward connectivity from a visual input region to a multimodal region during synchronous/congruent stimulation is common to our studies, the differences between the underlying models - left-hemispheric vs. bihemispheric, secondary vs. primary input regions, intrinsic modulation of all nodes vs. input nodes -precludes a detailed comparison.

\section{Attenuation of intrinsic S1 connectivity for mechanoreceptive signal processing}

We found intrinsic S1 connectivity to be reduced in CONGRUENT, the condition reflecting the illusory hand ownership feeling. Changes of intrinsic connections reflect local adaptation of neuronal populations connected horizontally or vertically within or between cortical layers \{Kiebel, $2007 \# 856\}$. As compared to EP source analysis alone $\{$ Zeller, $2014 \# 810\}$, the additional value of the DCM approach is that it combines a spatial forward model with a neurobiologically informed temporal forward model, describing the connectivity among sources \{Litvak, $2011 \# 320\}$. Our previous findings indicated that the source strength of mechanoreceptive signal processing in $\mathrm{S} 1$ is reduced when RHI is elicited. The present results extend these observations to indicate that the intrinsic connectivity is reduced in the local regional network of S1, thus indicating a profound modulation of the underlying local neuronal dynamics $\{$ Litvak, $2011 \# 320\}$. 
It is important to bear in mind that, from a unimodal, mechanoreceptive point of view, the three stimulation conditions are the same: Brushstrokes are repetitively applied to the skin of the fingers of the right hand. The REAL condition is the only condition where the signals provided by vision, touch and proprioception a priori coincide. It was, therefore, considered the reference condition. In contrast, the CONGRUENT condition poses a challenge to the brain. Even though the subject is aware of the fact that the seen hand is a surrogate, its position is plausible enough to allow the brain to consider it as its own hand. At the same time, the seen brushstrokes are felt synchronously at the corresponding skin area. Empirically, this degree of synchronicity and congruency appears highly unlikely to occur by chance. However, proprioceptive information about the position of the hand contradicts the simpler explanation that the seen hand and felt hand are the same. A Bayesian solution out of this dilemma might be to weight each of the ambiguous measurements by its relative precision \{O'Reilly, $2012 \# 809$;Brown, $2013 \# 323\}$. A reduction of the precision of ascending proprioceptive inputs by means of top-down modulation may decrease their influence on the resulting prediction \{Zeller, 2014 \#810;Feldman, 2010 \#390;Friston, 2007 \#386\}. The significant decrease of intrinsic connectivity of S1 in the CONGRUENT condition described here may indeed reflect reduced somatosensory precision, and is well in line with the underlying theory of predictive coding: A short-term surprise resulting from contradictory sensory inputs is avoided by top-down influence on the local processes at the somatosensory cortex $\{$ Apps, $2014 \# 830\}$. An attenuation of somatosensory precision may even be considered a necessary precondition paving the way for the feeling of bodily ownership of the rubber hand which would mostly succeed in the CONGRUENT, but fail in the INCONGRUENT condition. Of note, attenuation of proprioceptive precision does not imply attenuation of mechanoreceptive precision. Because proprioceptive and mechanoreceptive signals are processed independently, and because brush-stroke evoked potentials are likely to engage only mechanoreceptive afferents, 
reduction of intrinsic connectivity in S1 likely reflects a hierarchically higher stage, i.e. on where multisensory signals from somatosensory quality converge. This is in line with a growing body of evidence indicating that early sensory regions also participate in multisensory encoding \{ Kayser, 2015 \#861\} \{Rohe, 2015 \#862\}.

In conclusion, our findings suggest that interactions within the occipital-premotor network during the RHI may reflect hierarchical information exchange according to the principles of predictive coding. Representational changes in the ventral PMC as a result of bottom-up influences on this node may be an important precondition for the perception of limb ownership. They also appear to provide evidence for top-down attenuation of $\mathrm{S} 1$ related to somatosensory inputs that contextualizes somatosensory information during the illusory percepts. These findings provide a compelling insight into the functional anatomy of multisensory integration and the perception of oneself. 


\section{Acknowledgements}

Supported by departmental research funds. KF was funded by the Wellcome trust. We thank Vladimir Litvak for his valuable assistance in study design, data acquisition, and analysis. All authors declare that they have no conflict of interest. 


\section{Figure legends}

Figure 1: Schematic overview of the neuronal models and model families tested. (A) Basic neuronal models including premotor cortex (PMC) or parietal cortex (PC). (B) Model families as defined by the connections to be modulated. S1: postcentral gyrus; OC: occipital cortex; IM (curved green arrow): intrinsic modulation; FW (straight green arrow): forward connection; straight red arrow: backward connection; black left right arrow: lateral connection; blue arrow: input node. Models are presented in neurological convention (left is left).

Figure 2: Bayesian model selection. (A) Head-to-head comparisons of model families. Models with premotor cortex (PMC) vs. parietal cortex (PC); with vs. without intrinsic modulation in postcentral gyrus (S1) and occipital cortex (OC), respectively; with vs. without modulation of forward connections from S1 and OC, respectively. (B) Exceedance probabilities for each model separately. (C) Model posterior probability. Model No 6 exceeds all other tested models.

Figure 3: Trial-specific effects within (B) the winning model $\mathrm{N}^{\mathrm{o}} 6$. (A) The intrinsic connectivity of the left somatosensory cortex (S1) was lower for the CONGRUENT (CONG) than for the REAL condition, but comparable between INCONGRUENT (INCO) and REAL. (C) The forward (FW) connection from the left occipital cortex (OC) to the left premotor cortex (PMC) was stronger for CONG and INCO as compared to REAL. Significant differences between conditions are marked by an asterisk. The parametric effects are expressed as percentage changes from the average connectivity over conditions. 
Table 1: Definition of model families.

\begin{tabular}{ll|ll}
\hline Family & models No & Family & \multicolumn{1}{l}{ models No } \\
\hline PMC & $1-16$ & PC & $17-32$ \\
\hline S1 FW & odd numbers & without S1 FW & even numbers \\
\hline OC FW & $1,2,5,6,9,10,13,14$, & without OC FW & $3,4,7,8,11,12,15,16$, \\
& $17,18,21,22,25,26,29,30$ & & $19,20,23,24,27,28,31,32$ \\
& $5-8,13-16$, & & \\
\hline S1 IM & $21-24,29-32$ & & $17-4,9-12$, \\
& $9-16,25-32$ & without S1 IM & $1-25-28$ \\
\hline OC IM & & & \\
\hline
\end{tabular}

PMC, premotor cortex; S1, somatosensory cortex; OC, occipital cortex; FW, forward connection; IM, intrinsic modulation. 


\section{REFERENCES}

Gross, CL (2010). Die funktionelle Beteiligung verschiedener Hirnregionen in einer das Körperselbstgefühl täuschenden Illusion: Eine Läsionsstudie. [Functional participation of different brain regions in a body-ownerhip illusion- a lesion study.] Thesis.

Feldman H, Friston KJ (2010) Attention, uncertainty, and free-energy. Frontiers in Human Neuroscience 4:215. Kiebel SJ, Garrido MI, Friston KJ (2007) Dynamic causal modelling of evoked responses: the role of intrinsic connections. Neuroimage 36:332-345. 